\title{
Pediatric Aspects of the Michigan Polybrominated Biphenyl Contamination
}

\author{
MASON BARR, JR. \\ Department of Pediatrics, University of Michigan, Ann Arbor, Michigan 48109
}

Received January 25, 1979

\begin{abstract}
In 1973, Michigan cattle feed was accidentally contaminated with polybrominated biphenyls (PBB) and subsequently PBB has been found in the serum, body fat, and breast milk of most Michigan residents. Because of numerous complaints of ill health, a survey of Michigan farm children, the most heavily exposed group, was undertaken in 1976 to determine the nature and scope of the problem. Examination of the data from 292 Michigan children showed that the prevalence of symptoms was related to the quarantine status of the farm and to the method of invitation into the study. Serum PBB levels were related to the quarantine status of the farm but not to the method of invitation into the study. No significant effects of age or sex were found on the prevalence of symptoms or serum PBB levels, except that the teenage (13-16) males had somewhat higher PBB levels. Despite the frequent reporting of symptoms of ill health, physical examination failed to reveal any objective alterations that could be attributed to PBB. The most striking finding has been a statistically significant negative correlation between the prevalence of symptoms and the serum-PBB levels. So far, the reason for this negative correlation is unexplained.
\end{abstract}

\section{INTRODUCTION}

In 1973, polybrominated biphenyls (PBBs), a flame retardant, were accidentally mixed in cattle feed in Michigan (Carter, 1976; Kay, 1977). This set off an environmental contamination that reached the human population through its consumption of meat and milk from exposed herds. Although some data on toxicity of the chemically similar polychlorinated biphenyls (PCBs) had accumulated (Higuchi, 1976), at the time of the incident there was virtually no information on toxicity of PBBs. Because the PBBs appeared to be toxic to cattle (Jackson and Halbert, 1974), widespread concern developed about toxicity to humans and, in 1974-75, many claims of health problems among members of farm families began to surface. In November 1976, 40 months after the introduction of PBBs into the food chain, a health survey of Michigan farm families was undertaken. Preliminary analysis of some of the pediatric data from that study and reports of many of the findings in adults have been published elsewhere (Barr, 1978; Lilis et al., 1978; Valciukas et al., 1978; Anderson et al., 1978). This paper is concerned with the symptoms reported for 1976 (only), the findings on physical examination and serum-PBB levels in the children studied. This naper is being published not only to disseminate the results of the study but also to expose the study as a model for future studies of similar nature and to help other investigators avoid some of the pitfalls encountered.

\section{POPULATION STUDIED}

At the time of the study, it was known that most members of the Michigan population had detectable PBB in their blood and/or body fat and that farm fami- 
lies were certainly the most heavily exposed (Reizen, 1976; Brilliant et al., 1978; Anonymous, 1976). Farm families were invited to participate voluntarily in a study conducted by the Mount Sinai School of Medicine Environmental Sciences Laboratory and financed by the State of Michigan and the National Institute of Environmental Health Sciences. Informed consent was obtained from the parent/ guardian of each child before the examination, and the option to refuse any part of the testing was given.

Participants entered the study in several ways. The Michigan Department of Agriculture (MDA) provided a list of all farms where cattle had been tested for PBBs. Ninety-five children were from families invited randomly from the MDA farm list: 50 of these children were residents of quarantined farms (QR), ${ }^{1}$ and 45 were residents of nonquarantined farms (NQR). 'Twenty-seven children were from families invited because their farms were determined to be the most heavily contaminated $(\mathrm{QH})$. Thirty-six children had parents who were members of the Farmers' Advisory Council: 14 of these resided on quarantined farms (QF) and 22 on nonquarantined farms (NQF). One hundred and eighteen children were from families that entered the study by way of referral from doctors, lawycrs, or sclf: 67 were from quarantined farms (QS) and 51 from nonquarantined farms (NQS). Participants from the randomly selected farms were asked to give the names of people who had purchased meat or milk directly from the farm. These consumers were invited to participate in the study also. Thirty-five children were from families that had bought meat/milk directly from a quarantined farm (CQ) and 31 from families that had bought from a nonquarantined farm (CNQ). Because it appeared that most of the Michigan population had detectable PBB, an out-of-state, nonPBB-exposed population was sought as a control. Dairyfarm families from northcentral Wisconsin were asked to participate in a study of identical design and execution, and 72 Wisconsin dairyfarm children (WI) were examined.

\section{STUDY METHODS}

All Michigan children were examined November 4-10, 1976; and all Wisconsin children were examined March 25-26, 1977. Four examining physicians were used: three physicians (MB, GG, WR) examined children in Michigan and two (MB, MC) in Wisconsin. Attempts were made to adopt a standard terminology and consistency of method to reduce interobserver variability. Uncertainties in the physical examinations were checked by the senior pediatrician (MB). Prior to the physical examination, the parents of each child were asked to fill out a symptom review and to indicate whether or not the child had had any of a list of 75 symptoms (Table 1). If the symptom had been present, they were asked to indicate in which year(s), 1972-1976, the problem had occurred. Questions were also asked about the incidence of serious illness, hospitalizations, surgery, and accidents. Much additional historical information was collected at the time of the study; but, since that data will not be covered in this report, it is not itemized here.

At the time of examination, the questionnaire was reviewed by the physician and all positive responses were clarified by further questioning of the parent

' A farm was quarantined if the meat or milk produced contained $0.3 \mathrm{ppm}$ or more of PBB (fat basis). 
TABLE 1

SyMPTOMS INCLUDED IN THE QUESTIONNAIRE

\begin{tabular}{lll}
\hline Headache & Gum disease & Muscle cramps \\
Dizziness & Cough & Joint pains \\
Difficulty sleeping & Wheezing & Swollen joints \\
Irritability & Difficulty breathing & Skin rash \\
Easily upset & Bronchitis & Skin sensitive to sun \\
Nervousness & Pneumonia & Acne \\
Tiredness & Heart murmur & Change in skin color \\
Weakness & Heart disease & Dry skin \\
Loss of balance/clumsiness & Rapid pulse & Itching \\
Convulsions/fits/seizures & Blood-pressure problem & Skin sores/infection \\
Tremors/shakes & Vomiting & Boils \\
Numbness & Difficulty swallowing & Easy bruising \\
Loss of appetite & Abdominal pain & Slow healing of cuts \\
Eye redness & Heartburn & Excessive bleeding \\
Eye discharge & Constipation & Heavy perspiration \\
Vision problem & Diarthea & Hair loss \\
Glasses & Blood in stool & Hair changes \\
Multiple ear infections & Mucus in stool & Rough or abnormal nails \\
Hearing problems & Blood in urine & Slow growth (height) \\
Frequent colds & Dark (brown) urine & Slow growth (weight) \\
Runny nose & Urine infection & Behavior problems/school \\
Hay Fever & Pain on urination & Behavior problems/home \\
Allergies & Bedwetting & Trouble with friends/playmates \\
Sore throats & Frequent urination & Learning difficulties \\
Tooth decay/cavities & Broken bones & Poor school grades \\
\hline
\end{tabular}

and/or child. One of three codes was assigned to each positive response: (1) symptom apparently presented as a new complaint in the interval 1973-76; (2) symptom apparently present but dating from 1972 or before; and (3) symptom apparently not present as determined after further questioning. ${ }^{2}$ Of the original list of 75 symptoms presented to the parents, 14 were either combined with others on the list because of significant overlap (e.g., runny nose and colds) or dropped for want of positive responses (e.g., blood pressure problems, heart murmurs). Two other items, dental caries and acne, are treated here as physical findings rather than symptoms.

On completion of the history review, a general physical examination (excluding funduscopic, rectal, and vaginal exams) was performed; the results were rerorded on a physical-exam checklist. Children with abnormalities on physical examination or histories suggestive of particular illness were referred to their physicians for follow-up evaluation and treatment.

Laboratory studies included determination of serum PBB, in addition to other studies not reported here. Not all children had blood drawn for serum PBB; some parents, particularly of very young children, declined to have this done. The collection and analysis of serum PBB by electron-capture gas chromatography has been described by Wolff $e t$ al. (1978a).

\footnotetext{
"For example, if the parent had checked the item "frequent colds" but the child had four or fewer colds in the year, the response was disallowed.
} 
The data reported in this study were analyzed for the effects of several variables including: sex, age, method of invitation into the study, quarantine status of the farm, total number of symptoms reported and "verified" for 1976, and serumPBB level. Several factors entered into the decision to deal with the symptoms reported for 1976 only: major sources of $\mathrm{PBB}$ were apparently removed from the diet only by 1975 ; symptom data for the $1972-1976$ interval showed a relative plateau between 1975 and 1976; accuracy of recall is questionable, particularly over a 4-year period; and the physical examinations and serum-PBB levels were done in 1976. Comparison of the prevalence of the various symptoms among Michigan and Wisconsin children for the entire 1972-76 study period has been previously reported (Barr, 1978).

Total-symptom counts are used in this report. They are taken only to represent a crude measure of the parents' perception of the child's state of health. No distinction is made among the various symptoms as to relative importance or severity.

The symptom data and serum-PBB levels were not normally distributed. Because the variances of the PBBs and symptoms $(S+1)$ were generally proportional to the squares of the means of the various subgroupings analyzed, these data were log transformed to minimize the distortion of the means by the relatively fewer high serum-PBB levels and high total-symptom counts. Prevalence data were compared by $\chi^{2}$ analysis. Differences between means were tested by $t$ tests, and differences among means were tested by analysis of variance.

\section{RESULTS}

In a preliminary analysis of the data, it was found that Michigan parents reported a much higher prevalence of a wide variety of symptoms in their children than did Wisconsin parents (Barr, 1978). That analysis covered the prevalence of symptoms over the entire period studied $(73-76)$. The present study considers only those symptoms claimed for the year 1976. Except in a few identified instances, the only data considered are those from 292 children for whom serumPBB levels were obtained. Two additional children were omitted from the data analysis. One, a 4-year-old girl from a quarantined farm (self-invitation) had a serum PBB of $1625 \mathrm{ppb}$ and complained of four symptoms: frequent colds, anorexia, eye discharge, and otitis during 1976. Because this child's PBB level was of an order of magnitude ( $+3.8 \mathrm{SD}$ units above the mean) higher than the next several children, it was thought to distort the statistics disproportionately; and, therefore, this case was treated as an outlier and omitted from the calculations. The other child was a 15-year-old girl whose serum PBB was $0.2 \mathrm{ppb}$ and who reported no symptoms during 1976. However, because the records of her farm status and method of invitation into the study were not certain, it was decided to exclude her case from the study rather than misclassify it.

The Michigan and Wisconsin groups were compared for distribution between the sexes and for mean age, and no significant differences were found (Table 2). Intrafamily correlations have becn found for serum-PBB levels (Wolff et al., 1978b) and for symptomatology (unpublished data). The mean number of children per family studied did not differ significantly among the various groupings of the data employed here (e.g., Q, NQ: R, S, F, C, etc.). So, the entire population studied 
TABLE 2

Comparison of Michigan and Wisconsin Children Studied, by Age and Sex

\begin{tabular}{lccc}
\hline & $\begin{array}{c}\text { Males } \\
\text { age } \pm \text { SD }\end{array}$ & $\begin{array}{c}\text { Females } \\
\text { age } \pm \text { SD }\end{array}$ & $\begin{array}{c}\text { Both } \\
\text { age } \pm \text { SD }\end{array}$ \\
\hline Michigan & $9.5 \pm 4.6$ & $8.7 \pm 4.7$ & $9.3 \pm 4.2$ \\
Wisconsin & $n 168$ & $n 175$ & $n 343$ \\
& $10.8 \pm 4.0$ & $9.2 \pm 4.5$ & $10.1 \pm 4.2$ \\
& $n 40$ & $n 32$ & $n 72$ \\
\hline
\end{tabular}

was treated as individuals, and family units were ignored. The mean number of children per family was 2.5 (range 1-7) for the Michigan group and 2.6 (range 1-7) for the Wisconsin group.

The prevalence of symptoms among Michigan children as measured by totalsymptom count showed no significant differences in association with either age or sex (Table 3). The mean serum-PBB levels tended to be higher among males than among females in all age groups, but the difference was statistically significant $(P$ $<0.05$ ) only for the teenage $(13-16)$ group (Table 4$)$. Children in the 5 - to 8 -year range had significantly higher mean PBB levels than did children of other ages; this was true for both males and females (Table 4).

Both farm-quarantine status $(Q, N Q)$ and method of invitation into the study $(R$, $\mathrm{S}, \mathrm{F}, \mathrm{H}, \mathrm{C}) \mathrm{had}$ effects associated with the prevalence of symptoms. As anticipated, those who entered the study by self-referral $(S)$ complained of more symptoms regardless of the quarantine status of the farm (Table 5). Both groups (QS. NQS) had significantly more complaints than did the Wisconsin children. When the data were pooled and when the quarantine status of the farm was disregarded, self-invited children had about twice as many complaints as did other study participants $(P<0.001)$.

Children from the highly contaminated farms $(\mathrm{H})$ had the fewest complaints. In

TABLE 3

Mean Number of Symptoms Claimed for PBb-Exposed Chikuken, by Age and Sex"

\begin{tabular}{cccc}
\hline $\begin{array}{c}\text { Age } \\
\text { (yr) }\end{array}$ & Males & Females & Both \\
\hline $0-4$ & $3.3 \frac{12.9}{0.3}(14)$ & $3.0 \frac{10.3}{0.4}(19)$ & $3.1 \frac{11.1}{0.4}(33)$ \\
$5-8$ & $3.4 \frac{11.8}{0.6}(40)$ & $2.8 \frac{10.2}{0.3}(37)$ & $3.1 \frac{11.0}{0.4}(77)$ \\
$9-12$ & $2.3 \frac{7.0}{0.4}(37)$ & $3.2 \frac{10.0}{0.6}(44)$ & $2.8 \frac{8.5}{0.5}(81)$ \\
$13-16$ & $2.8 \frac{9.9}{0.3}(58)$ & $2.4 \frac{8.8}{0.2}(43)$ & 9.4 \\
& $2.9 \frac{9.7}{0.4}(149)$ & $2.8 \frac{9.7}{0.4}(143)$ & 0.3 \\
Total & $1(01)$ & 9.7 \\
\hline
\end{tabular}

"Geometric mean $\frac{+S D}{-S D}$ (number of children). 
TABLE 4

Serum-PBB Levels (ppb) of PBB-Exposed Children, by Age and Sex"

\begin{tabular}{ccccc}
\hline $\begin{array}{c}\text { Age } \\
(\mathrm{yr})\end{array}$ & Males & Females & $\begin{array}{c}\text { M vs F } \\
t\end{array}$ & Both \\
\hline $0-4$ & $3.93 \frac{25.34}{0.61}(14)$ & $2.11 \frac{9.15}{0.48}(19)$ & 1.073 & $2.75 \frac{14.30}{0.52}(33)$ \\
$5-8$ & $7.08 \frac{52.35}{0.95}(40)^{* *}$ & $4.32 \frac{25.60}{0.73}(37)^{* *}$ & 1.140 & $5.59 \frac{37.39}{0.83}(77)^{* *}$ \\
$9-12$ & $3.43 \frac{14.58}{0.80}(37)$ & $2.99 \frac{11.90}{0.75}(44)$ & 0.436 & $3.18 \frac{12.97}{0.78}(81)$ \\
$13-16$ & $3.45 \frac{13.29}{0.89}(58)$ & $1.87 \frac{7.87}{0.44}(43)$ & $2.199^{*}$ & $2.65 \frac{10.89}{0.64}(101)$ \\
Total & $4.23 \frac{21.67}{0.82}(149)$ & $2.72 \frac{12.69}{0.58}(143)$ & $2.375^{*}$ & $3.41 \frac{16.88}{0.68}(292)$ \\
\hline
\end{tabular}

"Geometric mean $\frac{+\mathrm{SD}}{-\mathrm{SD}}$ (number of children).

$* P<0.5$

${ }^{* *}$ Significantly higher than means for other age groups, $P<0.5$.

TABLE 5

Mean Number of Symptoms Claimed and Serum-PBB Levels (ppb) by Method of Invitation into The Study and Quarantine Status of The Farm

\begin{tabular}{|c|c|c|c|}
\hline $\begin{array}{c}\text { Farm/invite } \\
\text { status }\end{array}$ & $N$ & $\begin{array}{l}\text { Number of } \\
\text { symptoms }\end{array}$ & PBB \\
\hline QR & 42 & $2.1 \frac{6.6}{0.3}$ & $5.92 \frac{32.05}{1.10}$ \\
\hline QS & 52 & $4.2 \frac{12.7}{1.0} *$ & $5.11 \frac{30.45}{0.86}$ \\
\hline $\mathrm{QF}$ & 12 & $1.3 \frac{5.7}{0}$ & $10.02 \frac{28.16}{3.57}$ \\
\hline $\mathrm{QH}$ & 21 & $0.6 \frac{2.3}{0} *$ & $10.50 \frac{36.32}{3.03}$ \\
\hline NQR & 40 & $2.0 \frac{6.0}{0.3}$ & $2.68 \frac{9.69}{0.74}$ \\
\hline NQS & 48 & $6.0 \frac{15.2}{2.1} *$ & $1.62 \frac{4.74}{0.56}$ \\
\hline NQF & 19 & $4.0 \frac{12.5}{0.8} *$ & $0.89 \frac{2.20}{0.36}$ \\
\hline $\mathrm{CQ}$ & 31 & $1.6 \frac{6.5}{0}$ & $4.2 \frac{26.73}{0.73}$ \\
\hline CNQ & 27 & $4.1 \frac{15.3}{0.6} *$ & $1.94 \frac{7.96}{0.47}$ \\
\hline Total Michigan & 292 & $2.9 \frac{9.7}{0.4} *$ & $3.41 \frac{16.88}{0.68}$ \\
\hline
\end{tabular}


TABLE 5-Continued

\begin{tabular}{|c|c|c|c|}
\hline $\begin{array}{c}\text { Farm/invite } \\
\text { status }\end{array}$ & $N$ & $\begin{array}{l}\text { Number of } \\
\text { symptoms }\end{array}$ & PBB \\
\hline Total Wisconsin & 72 & $1.5 \frac{4.9}{0.1}$ & - \\
\hline All $\mathrm{R}$ farms & 82 & $2.1 \frac{6.3}{0.3}$ & $4.02 \frac{18.92}{0.86}$ \\
\hline All $S$ farms & 100 & $5.0 \frac{14.0}{1.4} *$ & $2.94 \frac{14.40}{0.60}$ \\
\hline All F farms & 31 & $2.7-\frac{9.3}{0.3}$ & $2.27 \frac{10.42}{0.50}$ \\
\hline All farms & 234 & $3.0 \frac{9.6}{0.5}$ & $3.56 \frac{17.22}{0.74}$ \\
\hline Consumers & 58 & $2.5 \frac{10.2}{0.1}$ & $3.01 \frac{15.99}{0.57}$ \\
\hline All Q & 158 & $2.2-\frac{7.7}{0.2}$ & $5.98 \frac{31.49}{1.14}$ \\
\hline All NQ & 134 & $3.9 \frac{12.1}{0.8} *$ & $1.79 \frac{6.14}{0.50}$ \\
\hline Total Michigan & 292 & $2.9 \frac{9.7}{0.4} *$ & $3.41 \frac{16.88}{0.68}$ \\
\hline Total Wisconsin & 72 & $1.5 \frac{4.9}{0.1}$ & \\
\hline
\end{tabular}

\begin{tabular}{|c|c|c|c|c|c|}
\hline \multirow[b]{2}{*}{ Comparison } & \multirow[b]{2}{*}{$d f$} & \multicolumn{2}{|c|}{ Symptoms } & \multicolumn{2}{|c|}{ PBB } \\
\hline & & $t$ & $\Gamma$ & $t$ & $\Gamma$ \\
\hline QR vs $N Q R$ & 80 & 0.218 & NSD & 2.385 & $<0.02$ \\
\hline QS vs NQS & 98 & -1.664 & NSD & 3.854 & $<0.001$ \\
\hline QF vs NQF & 29 & -2.175 & $<0.05$ & 6.867 & $<0.001$ \\
\hline CQ vs $\mathrm{CNQ}$ & 56 & -2.312 & $<0.025$ & 1.923 & $<0.10$ \\
\hline Q vs NQ & 290 & -3.596 & $<0.001$ & 6.932 & $<0.001$ \\
\hline Consumers vs farm & 290 & -0.750 & NSD & -0.713 & NSD \\
\hline & & $F$ & $P$ & $F$ & $P$ \\
\hline $\mathrm{QR}, \mathrm{QS}, \mathrm{QF}, \mathrm{QH}$ & $(3,123)$ & 9.403 & $<0.001$ & 1.330 & $>.10$ \\
\hline NQR, NQS, NQF & $(2.104)$ & 10.573 & $<0.001$ & 6.329 & $<.005$ \\
\hline R, S, F & $(2,210)$ & 12.948 & $<0.001$ & 1.762 & $>.10$ \\
\hline
\end{tabular}

Geometric mean $\frac{+\mathrm{SD}}{-\mathrm{SD}}$.

$* P<0.05$, compared with Wisconsin children.

fact, they had significantly fewer complaints than did the non-PBB-exposed Wisconsin children. Children from randomly selected farms (R) showed no differences in mean number of symptoms associated with the quarantine status of the farm and neither subgroup (QR, NQR) was significantly different from the Wisconsin children (Table 5). 
In the case of serum-PBB levcls, the method of invitation into the study appeared to have little bearing. For those children from quarantined farms, there were no significant differences $(P>0.10)$ among the various invitation-method groups. For those from the nonquarantined farms, the differences among the mean PBB levels, by invitation method, were significant $(\mathrm{NQR}>\mathrm{NQS}>\mathrm{NQF}, P<$ 0.005). However, when just the invitation method was compared (i.e., $R$ vs $S$ vs $\mathrm{F})$, the differences among the means were not significant $(P>0.10)$ although the trend $(\mathrm{R}>\mathrm{S}>\mathrm{F}$ ) was again seen (Table 5).

The quarantine status of the farm, either as residence or source of food, had a marked bearing on the serum-PBB level. Children from $Q$ farms had higher PBBs than did those from NQ farms. This was true within each invitation group $(R, S, F)$ and within the consumer $(C)$ group. In each case, the differences, $Q$ vs $N Q$, were significantly different (Table 5).

When the children were grouped by serum-PBB intervals, the mean age for each interval was virtually identical except for those with the highest PBB levels who were younger (Table 6). This was due to the significant excess of children in the 5to 8-year age group among those with serum PBB levels above $10 \mathrm{ppb}$ (Table 4). The mean age of children with no serum-PBB determinations was very much lower than the rest because samples were not taken from many of the youngest children by parental request. There were no significant differences in sex distribution among the PBB-interval groups. The majority of children with low PBB levels had a food source from a nonquarantined farm, and a majority of those with higher PBB levels had a food source from a quarantined farm (Table 6).

As the level of serum PBB increased, the mean number of reported symptoms decreased (Table 6). Such a phenomenon was suggested from the data in Table 5.

TABLE 6

Mean Age, Sex, Farm/Consumer Status, and Mean Number of Symptoms Claimed for 1976, by Serum-PBB Level Intervai.s

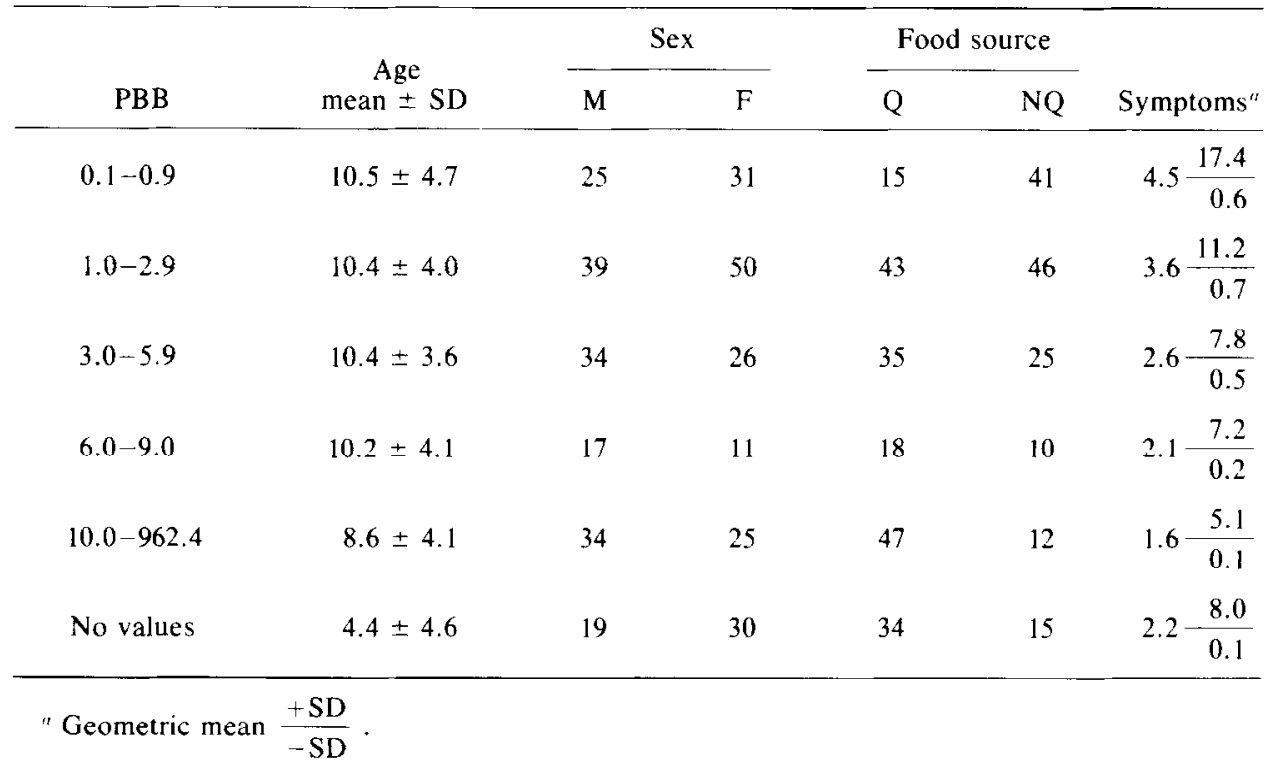


In that table, there is seen a reversal of the mean number of symptoms versus the mean serum PBB in the quarantined group as contrasted with the nonquarantined group.

If the basis of the classification is reversed, i.e., the children are grouped by the number of symptoms reported, the same negative correlation with serum PBB is found (Table 7). Among the Wisconsin sample 95\% of the children were reported to have five or fewer symptoms in 1976. If this is accepted as the "normal" experience, then attention should be directed to those Michigan children with six or more reported symptoms. Considering just those children with five or fewer symptoms, the differences in mean serum PBB are not striking. However, in the presence of six or more symptoms, the negative correlation is more marked.

Of the 59 symptoms analyzed, there was a pronounced tendency for those children with the symptom to have lower mean serum PBB than those without the symptom (Table 8 ). In the case of 35 symptoms, the differences in mean serum PBB were statistically significant, and the differences were of borderline significance for 4 other symptoms. In each case, the mean serum PBB was lower for children with the symptom. In only three instances were the mean serum PBBs of the children with a symptom higher than those without the symptom (pneumonia, urinary tract infection, and bedwetting), and in no case were the probabilities of significance less than $30 \%$.

The positive physical findings are summarized in Table 9 . This listing excludes minor skin abnormalities (nevi, hemangiomata, etc.), surgical scars, minor posi-

TABLE 7

Serum-PBB Levels (ppb) for Chil.dren with Various Numbers of Symptoms Claimed For $1976^{\prime \prime}$

\begin{tabular}{ccc}
\hline $\begin{array}{c}\text { Number of } \\
\text { symptoms }\end{array}$ & \multicolumn{1}{c}{ PBB } & $\begin{array}{c}\text { Number of } \\
\text { children }\end{array}$ \\
\hline 0 & $5.84 \frac{46.27}{0.74}$ & 69 \\
1 & $3.39 \frac{26.42}{0.99}$ & 36 \\
$2-3$ & $4.41 \frac{16.86}{1.15}$ & 62 \\
$4-5$ & $2.29 \frac{6.98}{0.75}$ & 31 \\
$6-9$ & $1.86 \frac{6.22}{0.56}$ & 37 \\
$10-19$ & $1.02 \frac{3.95}{0.26}$ & 39 \\
$20-39$ & $3.41 \frac{16.88}{0.68}$ & 292 \\
\hline
\end{tabular}

Geometric mean $\frac{+\mathrm{SD}}{-\mathrm{SD}}$. 
TABLE 8

Comparison of Mean Serum-PBB Levels (ppb) of Children with Particular SYMPTOMS VERSUS THOSE WITHOUT THE SYMPTOMS"

\begin{tabular}{|c|c|c|c|c|c|}
\hline \multirow[b]{2}{*}{ Symptom } & \multirow[b]{2}{*}{$N$} & \multicolumn{3}{|c|}{ PBB when symptom } & \multirow[b]{2}{*}{$t$} \\
\hline & & Pres & ent & Absent & \\
\hline \multicolumn{6}{|c|}{ A. Difference significant at $P<0.001$} \\
\hline Tiredness & 81 & 1.79 & $\frac{5.25}{0.60}$ & $4.36 \frac{23.83}{0.79}$ & -4.392 \\
\hline Irritability & 61 & $1.59-$ & $\frac{5.69}{0.44}$ & $4.17 \frac{21.02}{0.82}$ & -4.307 \\
\hline Recurrent headaches & 84 & $1.86-$ & $\frac{5.88}{0.59}$ & $4.34 \frac{23.58}{0.80}$ & -4.207 \\
\hline Muscle cramps & 68 & $1.83-$ & $\frac{7.91}{0.42}$ & $4.11 \frac{20.26}{0.83}$ & $-3,738$ \\
\hline Nervousness & 42 & 1.53 & $\frac{6.85}{0.34}$ & $3.90 \frac{18.92}{0.80}$ & -3.562 \\
\hline \multicolumn{6}{|c|}{ B. Difference significant at $P<0.01$} \\
\hline Anorexia & 27 & 1.27 & $\frac{4.59}{0.35}$ & $3.77 \frac{18.60}{0.76}$ & -3.418 \\
\hline Weakness & 32 & $1.42-$ & $\frac{3.68}{0.54}$ & $3.80 \frac{19.39}{0.76}$ & -3.344 \\
\hline Tremors & 12 & 0.78 & $\frac{2.05}{0.29}$ & $3.63 \frac{17.84}{0.73}$ & -3.327 \\
\hline Vertigo & 41 & $1.62-$ & $\frac{3.81}{0.68}$ & $3.85-\frac{20.24}{0.73}$ & -3.267 \\
\hline Otitis & 15 & 0.95 & $\frac{3.08}{0.29}$ & $3.65 \frac{17.95}{0.74}$ & -3.234 \\
\hline Dermatitis & 50 & $1.79-$ & $\frac{6.33}{0.50}$ & $3.89-\frac{19.90}{0.76}$ & -3.165 \\
\hline $\begin{array}{l}\text { School discipline } \\
\text { problem }\end{array}$ & 16 & 1.03 & $\frac{3.69}{0.28}$ & $3.65 \frac{17.93}{0.74}$ & -3.132 \\
\hline Xerodermia & 57 & $1.90-$ & $\frac{8.21}{0.44}$ & $3.92-\frac{19.49}{0.78}$ & -3.104 \\
\hline Trouble with peers & 20 & $1.22-$ & $\frac{4.27}{0.34}$ & $3.68 \frac{18.18}{0.74}$ & -3.014 \\
\hline Swollen joints & 14 & 1.03 & $\frac{4.08}{0.25}$ & $3.62 \frac{17.73}{0.73}$ & -2.905 \\
\hline Mood lability & 64 & $2.08-$ & $\frac{9.93}{0.43}$ & $3.91 \frac{19.11}{0.80}$ & -2.833 \\
\hline Dystrophic nails & 21 & 1.35 & $\frac{4.81}{0.38}$ & $3.66 \frac{18.18}{0.73}$ & -2.775 \\
\hline Eye discharge & 20 & $1.36-$ & $\frac{4.54}{0.40}$ & $3.65 \frac{18.17}{0.73}$ & -2.681 \\
\hline Learning difficulty & 40 & $1.24-$ & $\frac{4.58}{0.33}$ & $3.61 \frac{17.86}{0.73}$ & -2.628 \\
\hline
\end{tabular}


TABLE 8-Continued

\begin{tabular}{|c|c|c|c|c|}
\hline \multirow[b]{2}{*}{ Symptom } & \multirow[b]{2}{*}{$N$} & \multicolumn{2}{|c|}{ PBB when symptom } & \multirow[b]{2}{*}{1} \\
\hline & & Present & Absent & \\
\hline Open skin sores & 27 & $1.59 \frac{5.66}{0.44}$ & $3.68 \frac{18.46}{0.73}$ & -2.622 \\
\hline \multicolumn{5}{|c|}{ C. Difference significant at $P<.05$} \\
\hline Abdominal pain & 50 & $2.02 \frac{8.12}{0.50}$ & $3.80-\frac{19.21}{0.75}$ & -2.575 \\
\hline Poor weight gain & 14 & $1.18 \frac{5.49}{0.25}$ & $3.60-\frac{17.58}{0.73}$ & -2.572 \\
\hline Frequent colds & 64 & $2.20 \frac{8.97}{0.53}$ & $3.85 \frac{19.70}{0.75}$ & -2.506 \\
\hline Clumsiness & 25 & $1.63 \frac{7.30}{0.36}$ & $3.65-\frac{18.43}{0.74}$ & -2.435 \\
\hline Insomnia & 21 & $1.51 \frac{4.75}{0.48}$ & $3.63 \frac{18.24}{0.72}$ & -2.434 \\
\hline Pruritis & 44 & $2.01 \frac{9.40}{0.42}$ & $3.74 \frac{18.43}{0.76}$ & -2.405 \\
\hline Heavy sweating & 28 & $1.73 \frac{7.07}{0.42}$ & $3.66 \frac{18.22}{0.73}$ & -2.370 \\
\hline Hair changes & 16 & $1.38 \frac{5.59}{0.34}$ & $3.59 \frac{17.74}{0.72}$ & -2.339 \\
\hline $\begin{array}{l}\text { Home discipline } \\
\text { problem }\end{array}$ & 31 & $1.82 \frac{8.58}{0.38}$ & $3.67 \frac{18.04}{0.74}$ & -2.329 \\
\hline Persisting cough & 40 & $1.98 \frac{8.54}{0.46}$ & $3.71 \frac{18.52}{0.74}$ & -2.319 \\
\hline Dysphagia & 9 & $1.14-\frac{4.20}{0.30}$ & $3.53 \frac{17.45}{0.71}$ & -2.100 \\
\hline $\begin{array}{l}\text { Frequent pharyngitis } \\
\text { (>4/year) }\end{array}$ & 60 & $2.33 \frac{10.48}{0.51}$ & $3.76 \frac{18.85}{0.74}$ & -2.078 \\
\hline Poor school grades & 17 & $1.60 \frac{4.93}{0.51}$ & $3.57 \frac{17.95}{0.71}$ & -2.019 \\
\hline Easy bruising & 15 & $1.55 \frac{6.06}{0.39}$ & $3.56 \frac{17.66}{0.71}$ & -1.976 \\
\hline Mucus in feces & 12 & $1.41 \frac{5.26}{0.37}$ & $3.54 \frac{17.57}{0.71}$ & -1.968 \\
\hline \multicolumn{5}{|c|}{ D. Difference not significant $0.10>P>0.05$} \\
\hline Urinary frequency & 26 & $1.91 \frac{11.36}{0.32}$ & $3.61 \frac{17.39}{0.74}$ & -1.937 \\
\hline Dyspnea & 16 & $1.62 \frac{4.75}{0.55}$ & $3.56 \frac{17.91}{0.70}$ & -1.919 \\
\hline Numbness & 24 & $1.91 \frac{9.05}{0.40}$ & $3.59 \frac{17.72}{0.72}$ & -1.852 \\
\hline Diarrhea & 32 & $2.13 \frac{11.90}{0.38}$ & $3.61-\frac{17.50}{0.74}$ & -1.766 \\
\hline
\end{tabular}


TABLE 8-Continued

\begin{tabular}{|c|c|c|c|c|}
\hline \multirow[b]{2}{*}{ Symptom } & \multirow[b]{2}{*}{$N$} & \multicolumn{2}{|c|}{ PBB when symptom } & \multirow[b]{2}{*}{$t$} \\
\hline & & Present & Absent & \\
\hline \multicolumn{5}{|c|}{ E. No significant difference $P>0.10$} \\
\hline Conjunctivitis & 26 & $2.10 \frac{7.58}{0.58}$ & $3.57-\frac{18.09}{0.70}$ & -1.618 \\
\hline Excessive bleeding & 15 & $1.85 \frac{3.21}{1.06}$ & $3.52 \frac{18.01}{0.68}$ & -1.526 \\
\hline Visual difficulty & 19 & $2.00-\frac{7.69}{0.51}$ & $3.54-\frac{17.72}{0.70}$ & -1.506 \\
\hline Hair loss & 12 & $1.79 \frac{7.65}{0.41}$ & $3.50-\frac{17.40}{0.70}$ & -1.430 \\
\hline Constipation & 20 & $2.11 \frac{7.02}{0.63}$ & $3.53-\frac{17.87}{0.69}$ & -1.391 \\
\hline Arthralgia & 67 & $2.74 \frac{12.70}{0.59}$ & $3.64-\frac{18.31}{0.72}$ & -1.277 \\
\hline Dysuria & 9 & $1.78 \frac{6.85}{0.46}$ & $3.48-\frac{17.32}{0.69}$ & -1.235 \\
\hline Slow healing & 11 & $2.11 \frac{7.77}{0.57}$ & $3.47 \frac{17.36}{0.69}$ & -1.017 \\
\hline Heartburn & 18 & $2.50 \frac{9.06}{0.69}$ & $3.48 \frac{17.54}{0.68}$ & -0.844 \\
\hline Vomiting & 22 & $2.61 \frac{11.10}{0.61}$ & $3.48 \frac{17.46}{0.69}$ & -0.818 \\
\hline
\end{tabular}

F. Insufficient positive responses for meaningful analysis: Bedwetting (over 4 years old, 8), hay fever/allergies (7), blood in feces (7), bronchitis (6), urinary tract infection (6), photosensitivity (5), boils (5), pneumonitis (4), tachycardia (4), slow height gain (3).

"Geometric mean $\frac{+S D}{-S D}$.

TABLE 9

Findings on Physical Examination of Wisconsin and Michigan Child dren, Quarantine Status of Food Source, and Mean Serum PBB

\begin{tabular}{|c|c|c|c|c|c|}
\hline \multirow[b]{2}{*}{ Finding } & \multirow[b]{2}{*}{$\begin{array}{c}\text { Wisconsin } \\
n\end{array}$} & \multicolumn{4}{|c|}{ Michigan } \\
\hline & & $\begin{array}{c}\text { Total } \\
n\end{array}$ & $\begin{array}{l}\mathrm{Q} \\
n\end{array}$ & $\begin{array}{c}\mathrm{NQ} \\
n\end{array}$ & $\begin{array}{c}\mathrm{PBB}^{\prime \prime} \\
\mathrm{ppb}\end{array}$ \\
\hline Upper respiratory infection & 13 & 41 & 21 & 20 & $3.64 \frac{19.22}{0.69}$ \\
\hline Functional heart murmur & 6 & 29 & 14 & 15 & $3.46 \frac{19.51}{0.62}$ \\
\hline Macular skin rash & 9 & 25 & 16 & 9 & $3.36 \frac{25.34}{0.45}$ \\
\hline Folliculitis & 4 & 20 & 9 & 11 & $2.49 \frac{14.37}{0.43}$ \\
\hline
\end{tabular}


TABLE 9-Continued

\begin{tabular}{|c|c|c|c|c|c|}
\hline \multirow[b]{2}{*}{ Finding } & \multirow[b]{2}{*}{$\begin{array}{c}\text { Wisconsin } \\
\text { In }\end{array}$} & \multicolumn{4}{|c|}{ Michigan } \\
\hline & & $\begin{array}{c}\text { Total } \\
n\end{array}$ & $\begin{array}{l}\mathrm{Q} \\
n\end{array}$ & $\begin{array}{c}\mathrm{NQ} \\
n\end{array}$ & $\begin{array}{r}\mathrm{PBB}^{\prime \prime} \\
\mathrm{ppb}\end{array}$ \\
\hline Otitis media & 8 & 19 & $6^{b}$ & $13^{\prime \prime}$ & $2.82 \frac{15.85}{0.50}$ \\
\hline Tenderness extremities & 0 & 16 & 8 & 8 & $4.61 \frac{23.21}{0.92}$ \\
\hline Dystrophic nails & 0 & 15 & 9 & 6 & $2.65 \frac{18.86}{0.37}$ \\
\hline Palpable liver" & 1 & 15 & 6 & 9 & $1.18 \frac{7.32}{0.19}$ \\
\hline Dry. scaly skin & 3 & 14 & $4^{\prime t}$ & $10^{4}$ & $2.36 \frac{9.39}{0.60}$ \\
\hline Atopy" & 3 & 12 & 6 & 6 & $4.32 \frac{13.92}{1.34}$ \\
\hline Obesity, marked & 5 & 10 & 5 & 5 & $2.13 \frac{5.35}{0.85}$ \\
\hline Abnormal neurologic" & 5 & 7 & 2 & 5 & $2.12-\frac{8.92}{0.50}$ \\
\hline Conjunctivitis & 2 & 7 & 2 & 5 & $2.28 \frac{4.52}{1.15}$ \\
\hline Enlarged, tender nodes & 2 & 7 & 3 & 4 & $2.08 \frac{15.34}{0.28}$ \\
\hline Thyromegaly & 2 & 6 & 5 & 1 & $6.74 \frac{37.28}{1.22}$ \\
\hline Elevated blood pressure & 0 & 4 & 4 & 0 & $13.62 \frac{71.55}{2.59}$ \\
\hline All children & 72 & 343 & 193 & 149 & $3.41 \frac{16.88}{0.68}$ \\
\hline
\end{tabular}

Other findings (frequency): Abdominal tenderness (M5), warts (M6, W3), tibial nodule (M3), neck tenderness (M4), eye-muscle dysfunction (M4), periodontal disease (M2), mottled enamel (M3). pigmented buccal lesion (M1), tinea corporis (MI), tachypnea (M1), preauricular skin tag (M1), deviated nasal septum (M1), transposition of the great vessels (M1), suspected ventricular septal defect (M1), supernumerary tooth (W1), cheilitis (W1), impetigo (W1), brittle hair (M1).

"Geometric mean $\frac{+\mathrm{SD}}{-\mathrm{SD}}$.

${ }^{b} \chi^{2} 4.041,0.05>P>0.025$.

- See text for more detail.

" $\chi^{2} 3.503,0.10>P>0.05$.

" Atopy includes: asthma, eczema, and chronic allergic nasal congestion. 
tional deviations of the extremitics, and signs clearly due to trauma. There were no statistically significant differences in the prevalence of findings between the Michigan and Wisconsin children. Nor were there any significant associations, positive or negative, between abnormalities and serum-PBB levels among the Michigan children.

The finding of tenderness to pressure over the extremities was restricted to the Michigan children, but the difference in prevalence is not statistically significant $\left(\chi^{2} 2.132, P>0.10\right)$. Since the tenderness was found in various locations in the arms and legs, it is difficult to regard the observations as significant. Another finding restricted to the Michigan children was dystrophy of the nails, but again the difference in prevalence was not statistically significant. Also, the form of changes in the nails varied from thin and brittle to thick and rough, so the term dystrophic nails is not to be taken as a description of a specific entity. The livers of 16 children $(15 \mathrm{M}, 1 \mathrm{~W})$ were palpated $1-3 \mathrm{~cm}$ below the costal margin. Except in the case of 1 child with definite hepatosplenomegaly and suspected ventricular septal defect, all of the livers were thought to be of normal size, and most of the livers palpated below the costal margin were found in children less than 3 years old.

Abnormalities on neurological examination were infrequent, and no common pattern of abnormality was found. The abnormalities included: mild athetosis $(2 \mathrm{~W})$, intention tremor $(1 \mathrm{~W})$, minimal or absent associated arm swing with ambulation ( $2 \mathrm{~W})$, hyperkinesis $(2 \mathrm{M})$, depression (1M), abnormal Romberg test (2M), chronic anxiety state $(1 \mathrm{M})$, and sensorineural hearing loss (hereditary, $2 \mathrm{M})$. The blood pressures of four Michigan children were persistently elevated (systolic > $140 \mathrm{~mm} \mathrm{Hg}$ : diastolic $>100 \mathrm{~mm} \mathrm{Hg}$ ). In only one case was the elevation thought to be significant; the others were systolic elevations most likely due to anxiety.

Because acne is an age-related condition and because the halogenated hydrocarbons are known to produce chloracne, special attention was directed to those children with acne. The earliest age at which acne was found was 10 years in the Michigan sample and 11 years in the Wisconsin sample. The prevalence of acne was not significantly different among the 10- to 11-year-old Michigan children (15.6 vs $27.3 \% \chi^{2} 0.015$ ) or among the 10 - to 16 -year-old Michigan children (36.2 vs $41.9 \% \chi^{2} 0.260$ ) than among their Wisconsin counterparts. It was found that the Michigan 10 to 11 year olds with acne had a significantly higher mean serum PBB (7.41 ppb) than the 10 to 11 year olds without acne $(2.30 \mathrm{ppb})(t 2.609,43 d f, P<$ 0.02). However, considering the entire sample of children at risk for acne, the mean serum PBB of those with acne $(2.90 \mathrm{ppb})$ was not significantly different from those without acne ( $2.59 \mathrm{ppb})$. None of the children examined was found to have chloracne: all appeared to have the ordinary inflammatory variety of adolescent acne.

In the case of active dental caries, there was no significant difference in prevalence between the Michigan and Wisconsin children ( $\chi^{2}$ 0.002). Among the Michigan children, those with unrepaired caries had a significantly lower mean serum PBB (1.61 vs $3.69 \mathrm{ppb}, t 2.629,290 \mathrm{df}, P<0.01)$ and were significantly more likely to have a nonquarantined farm as a food source ( 13.4 vs $6.2 \%, \chi^{2} 4.333, P<0.05$ ) than those with no active caries. Whether these relationships are similar to the 
inverse correlation of PBB with symptoms or are only an effect of variation in health-care patterns is not certain.

\section{DISCUSSION}

Political and social implications of the PBB incident make it necessary to state that the results of this study do not indicate that PBBs are beneficial. There is no evidence whatsoever that Michigan children contaminated with PBBs are better off than the unexposed Wisconsin children. The question is whether the Michigan children are worse off for their exposure.

It must be emphasized that this study was performed 3 years after the onset of exposure. Neither the actual time of onset nor the duration of exposure have been determined for any of the children. However, it is reasonably estimated that significant exposure had ceased about 9-12 months before the study and the assumption is made that this interval allowed equilibration of PBB storage to take place. Although PBB has been shown to be hepatotoxic (Aftosmis et al., 1972), no significant abnormalities of liver functions could be demonstrated among a sampling of the teenagers (unpublished data). This would indicate that either no liver damage was produced or that sufficient time had elapsed to allow for repair of acute damage. The half-life of PBB stored in body fat appears to be very long, measured in months or perhaps years, and levels are unlikely to have diminished to any appreciable extent during the interval between exposure and study (Matthews et al., 1977). Thus, it is assumed that the study is measuring subacute effects of PBB contamination.

Although the data reported here represent a point prevalence study of 1976 symptoms, information was gathered on the prevalence of symptoms over the entire exposure period. It is apparent that the prevalence of symptoms began to rise among the Michigan farm children in 1974 and continued to rise until 1975 (Barr, 1978). While there was a subjective impression of a decrease in reported symptoms from early to late 1976, the data recorded here are for symptoms occurring at any time in 1976 and these symptoms were not appreciably fewer $\mathrm{J}$ different than those reported to have occurred in 1975. The time-prevalence curves for children from quarantined farms were not demonstrably different from the curves for children from nonquarantined farms. This indicates that a hypothesis of earlier exposure and earlier recovery for quarantined-farm children is not tenable.

Because PBBs are lipotrophic substances, it is presumed that the clinically most important measurement is the body-fat level and calculation of total body burden of PBBs. However, obtaining fat biopsies from children, particularly in such large numbers in the short time available, was thought to be not feasible. Although fat/serum PBB ratios have been found to vary (Brilliant et al., 1978; Landrigan et al., 1978; Meester and McCoy, 1976), there is a reasonably constant relationship that permits some inference about fat levels from knowledge of serum levels. Also it would seem reasonable that fat/blood ratios of PBBs would have stabilized by 1976, at which time the major new sources of PBB had been eliminated from the diets of Michigan residents. Because information is not available on the actual amount of PBBs ingested and absorbed by the study participants, certain answers must be inferred from the data indirectly on the basis of the serum-PBB levels. 
Another confounding variable is the nature of the chemical. PBB is not a single compound but a mixture of biphenyls with varying degrees of bromine saturation and varying molecular configurations. Each of these isomers differs from others in the mixture in its absorption, metabolism, excretion, and toxicity. Current evidence suggests that the major constituent of the mixture $\left(2,4,5,2^{\prime}, 4^{\prime}, 5^{\prime}\right.$ hexabromobiphenyl), the constituent that is measured chromatographically, is not the toxic component (Hass et al., 1978). Furthermore, it has been demonstrated that by passage through cattle prior to consumption by another animal, the toxicity of PBBs is enhanced (Aulerich and Ringer, 1979). This is presumably due to differential increases in the toxic components either by quantitative reduction of less toxic components or by metabolism of relatively inert forms to toxic metabolites.

The children in this study were not exposed to the original industrial mixture of PBBs but rather to PBBs that had been cycled through cattle, swine, and/or poultry. Presumably, however, all of the Michigan children had been similarly exposed to "recycled" PBBs and there is no a priori reason to suppose that some contaminated beef was more toxic than other contaminated beef other than as determined by the amount of total PBBs present. Thus it is assumed that measurement of the major, albeit relatively nontoxic, component of the PBB mixture provides an accurate basis for comparing the toxic exposures among the children.

It is presumed that a number of the findings of this study reflect the diets of the participants. At the outset, it was expected that farm residents who consumed the contaminated meat and milk from their subsequently quarantined herds would have received the highest doses of PBBs. That this notion was substantially correct is evident from the fact that the serum PBB levels of quarantined-farm children were significantly higher than those of nonquarantined-farm children. Studies in adults have shown that husbands, on the average, have higher serum PBB levels than their wives (Wolff et al., 1978b) This is presumed to be a reflection of the greater quantity of food consumed by the adult male. Among children, the sex differences, with males having the higher levels, were seen at all ages; but the differences were not statistically significant until teenage. This finding would seem to fit well with the concept that food consumption is very similar between the sexes in childhood and diverges toward greater consumption by males during adolescence.

Somewhat puzzling is the finding of significantly higher mean serum-PBB level in the 5- to 8-year-old age group. These children are too old to have been exposed to $\mathrm{PBB}$ in utero or, to any significant degree, from ingestion of contaminated breast milk. Nor are 5-8 year olds renowned for large food intake.

If growth rates at the time of presumed exposure are considered, a possible explanation of the higher PBB levels among the 58 ycar olds is apparent. The infants $(0-4$ years) may be characterized both by their limited exposure (duration and nature of diet) and by their rapid growth. The 5-8 year olds were potentially exposed to PBB-contaminated food during the entire 1973-75 period and their growth rates are relatively slow. The $9-12$ year olds also would have received a full exposure but, at least among the older ones of the group, their growth rates are accelerating. The teenagers (13-16 years) are in a phase of rapid growth. Rapid growth requires an increase in food consumption and thus a risk of an absolute 
increase in the consumption of PBBs. Unless concentration of the contaminant in the food is high enough to sustain saturation of the fat depot, accretion of body mass will dilute the concentration of PBBs in the fat depot, despite the fact that total body content of the contaminant increases. Unfortunately there are insufficient data on the quantitative and qualitative aspects of diet among children of this age to allow anything more than the framing of a very general hypothesis about the relation of growth rates and the accretion-concentration of dietary contaminants.

Aside from some relatively minor puzzles, the findings in regard to PBB levels were in line with what had been predicted before the data were gathered. On the other hand, the data regarding symptoms yielded some major surprises. Symptoms are at best an elusive quantity; the investigator can never be really sure that the complaint existed, and in many cases there is no confirming physical evidence, even at the time of the complaint. Additional problems are imposed when it is the parent, not the sufferer, who is claiming the symptom. Symptoms also vary in severity and frequency; and, of course, particular symptoms are of varying significance to the child, parent, and physician, both in regard to discomfort and as indicators of significant ill health.

In this study, an attempt was made to weed out the trivial and incorrect reporting of symptoms and to discount from consideration any symptoms that antedated possible exposure to PBB. A very few parents reported that their children had many symptoms which the examiner felt confident had never truly existed. In most cases, the exclusion of reported symptoms was minor. If the issue was uncertain, to include or exclude, the parents were given the benefit of the doubt and the positive response was included. The most frequent reason for disallowing a reported symptom was a denial by the child of ever having such a complaint. Some inaccuracy in reporting was thought to be due to faulty recall of events and confusion in the larger families over which child had which symptoms.

An opposite problem can occur when a child has had a symptom, but the parent is unaware or denies it. For the latter possibility, the parents were asked to check the item "no" to indicate definitely that the symptom had not been present. Despite this, there is evidence that some denial of symptoms took place. No truly effective way of distinguishing true negatives and false negatives was found.

Of the Michigan children studied, the parents of about one-third appeared to believe that their children's health had gotten worse, while the parents of twothirds thought their children's health had not changed either way between 1972 and 1976 (Barr, 1978). Although it is not quantifiable and cannot count in the analysis of data, the impression of each of the examiners was of a group of people who were patently honest and sincere in their belief that the health of their children had deteriorated in the 1973-76 interval.

Despite the problems inherent in the symptom data, the total-symptom count appeared to be a relatively accurate portrayal of how the parents perceived the health status of their children. Because it had been expected that, if PBB were harmful to human health, symptoms would be directly correlated with serum-PBB levels, the finding of a statistically significant inverse correlation was puzzling, to say the least. The fact that children with a nonquarantined farm as a food source had appreciably more symptoms than those whose food source was a quarantined farm has been commented on previously (Barr, 1978). Before the results of the 
serum-PBB determinations were available, it was postulated that residents of quarantined farms might have been warned of eating the contaminated food and, thus, have restricted their PBB intake. In an opposite manner, those from nonquarantined farms, with the assurance from authorities that PBB was not harmful, would have continued to eat food that was contaminated, albeit at lower levels, and continued to add PBBs to their bodies. However, the results of the serumPBB determinations show that this speculation was not the case, at least to any appreciable extent. Analysis of the data for individual symptoms shows the inverse association betwcen the symptom's presence and serum $\mathrm{PBB}$ in virtually every case.

Despite the frequent reporting of symptoms of ill health among the PBBexposed children that were studied, physical examination failed to reveal any objective alterations that could be attributed to PBB. An independent study of 3to 5-year-old Michigan dairy-farm children by Weil et al. (1977) has confirmed this. Despite the failure to find any physical signs of toxicity, Seagull has reported very disquieting evidence that the performance of $\mathrm{PBB}$-exposed children may be adversely affected (Seagull, 1978). Children with fat PBBs above 100 ppb performed more poorly on four of five subtests of the McCarthy scales.

The findings of this study in relation to the correlations between symptoms and serum-PBB levels appear to testify against the notion of toxicity. While the evidence that has been presented does not indicate that PBBs are beneficial to health, the almost totally consistent negative relation between symptoms and serum PBB is at odds with a dose-response concept of toxicity. Why there are significant negative correlations between either total-symptom counts or individual symptoms and serum-PBB levels is by no means clear.

The question now is whether this inverse correlation between symptoms and serum PBB has a biological, social, statistical, or epidemiological basis. That question cannot be fully answered yet; but a fuller analysis of the data in hand, now in progress, should help resolve it.

The finding that children from the most highly contaminated farms reported fewer complaints than did the unexposed Wisconsin children suggests that some of those people who knew they were highly exposed may have consciously or unconsciously denied having symptoms. A combined process of denial by those with high serum-PBB levels and overreporting by those with low serum-PBB levels could explain the negative correlation observed between PBB levels and symptoms. However, the vast majority of families studied had no prior knowledge, from testing of their blood or fat, of what their PBB levels were. Therefore, it is difficult to imagine that processes of denial and overreporting of symptoms could have worked effectively enough to produce the consistently negative correlations found.

In the early stages of investigation, it had been postulated that anxiety and social/economic dislocation caused by the contamination, toxicity, and quarantining of the cattle could precipitate an increased prevalence of health complaints. If this were a major factor, it would be expected that residents of quarantined farms, who presumably experienced the most hardship, would report more ill health; such was not the case. Also among consumers, who experienced no social or economic disruption, those who consumed meat/milk from quarantined farms 
reported fewer symptoms in the face of higher PBB levels than did those who got food from nonquarantined farms.

The social and political considerations of the Michigan PBB incident cannot be ignored in the study of potential toxicity. As has been suggested in the case of the Seveso-dioxin incident, measures taken or not taken to control and investigate the contamination may have a very important effect on peoples' perception of their health (Whiteside, 1978). Factors such as guilt, embarrassment, and disruption of life patterns by governmental agencies may actually foster the denial of symptoms. On the other hand, underattention to exposed people may serve to increase the number of complaints of ill health. It is certainly possible that what has been found thus far is less an effect of PBBs than an effect of the PBB incident.

In the investigation of toxicity, there are considerations other than that of the type of toxicity addressed here. This study has not dealt with the question of more subtle potential effects on growth and performance among exposed children. Nor can it answer questions about long-term effects of PBBs such as a future increase in the incidence of malignancies or a decrease in reproductive performance. These are problems of continuing concern to many PBB-exposed people and their physicians.

\section{ACKNOWLEDGMENTS}

Deep gratitude is expressed to the following people for their help with the study and their helpful comments on this report: Henry A. Anderson, Irving J. Selikoff, Walter J. Rogan, Robert C. Kelsch, Helen S. Barr. Genevieve Go, and Mitchell Cohen. Appreciation is also due to many members of the Environmental Sciences Laboratory of the Mount Sinai Medical Center for their essential roles in getting the study done.

\section{REFERENCES}

Aftosmis, J. G. et al. (1972). Toxicology of polybrominated biphenyls. II. Skin, eye and inhalation toxicity and an acute test method for evaluating hepatotoxicity and accumulation in body fat. Toxicol. Appl. Phurmacol. 22, 316-317.

Anderson, H. A., Lilis, R., Selikoff, I. J., Rosenman, K. D., Valciukas, J. A., and Freedman, S. (1978). Unanticipated prevalence of symptoms among dairy farmers in Michigan and Wisconsin. Environ. Health Perspect. 23, 217 - 226.

Anonymous. (1976). Is PBB a human health hazard? Mich. Med. 75, 560.

Aulerich, R. J., and Ringer, R. K. (1979). Toxic effects of dietary polybrominated biphenyls on mink. Arch. Fniton. Contam. Toxicol. 8, 487-498.

Barr, M. (1978). Pediatric health aspects of PBBs. Environ. Health Perspect. 23, 291-294.

Brilliant, L. B., Van Amburg, G., Isbister, J., Humphrey, H., Wilcox, K., Eyster, J., Bloomer, A. W., and Price, H. (1978). Breast-milk monitoring to measure Michigan's contamination with polybrominated biphenyls. Lancet 2, 643-646.

Carter, L. J. (1976). Michigan's PBB incident: Chemical mix-up leads to disaster. Science 192. $240-243$.

Hass, J. R., McConnell, E. E., and Harvan, D. J. (1978). Chemical and toxicologic evaluation of Firemaster BP-6. J. Agr. Food Chem. 26, 94-99.

Higuchi. K., Ed. (1976). PCB Poisoning and Pollution." Academic Press, New York.

Jackson, T. F., and Halbert, F. L. (1974). Toxic syndrome associated with the feeding of polybrominated biphenyl-contaminated protein concentrate to dairy cattle. J. Amer. Vet. Med. Ass. 165 , $437-439$.

Kay, K. (1977). Polybrominated biphenyls (PBB) environmental contamination in Michigan, 1973-1976. Environ. Res. 13, 74-93.

Landrigan, P. J., Wilcox. K., and Silva, J. (1978). "Cohort Study of Michigan Residents Exposed to 
Polybrominated Biphenyls: Epidemiological and Immunological Findings." Paper presented at New York Academy of Sciences, N. Y., June 24, 1978.

Lilis, R., Anderson, H. A., Valciukas, J. A., Freedman, S., and Selikoff, I. J. (1978). Comparison of findings among residents on Michigan dairy farms and consumers of produce purchased from these farms. Environ. Health Perspect. 23, 105-109.

Matthews, H. B., Kato, S., Morales, N. M., and Tuey, D. B. (1977). Distribution and excretion of $2,4,5,2^{\prime}, 4^{\prime}, 5^{\prime}$-hexabromobiphenyl, the major component of Firemaster BP-6. J. Toxicol. Environ. Health 3, 599-605.

Meester, W. D., and McCoy, D. J. (1976). "Human Toxicology of Polybrominated Biphenyls." Paper presented at American Academy of Chemical Toxicology, Seattle, Wash., August 4, 1976.

Reizen, M. S. (1976). "Letter to Michigan Physicians, August 26, 1976." Michigan Department of Public Health, Lansing, Mich.

Seagull, E. A. W. (1978). "Developmental Abilities of Children Exposed to Polybrominated Biphenyls." Press release from the Michigan Department of Public Health, Lansing, Mich., September 14, 1978.

Selikoff, I. J.. Anderson, H. A., Smith, H., and Calhoon, F. (1978). "A Survey of the General Population of Michigan for Health Effects of PBB Exposure: Interim Report (2/1/78-9/15/78)." Submitted to Michigan Department of Public Health, Lansing, Mich.

Valciukas, J. A., Lilis, R., Wolff, M. S., and Anderson, H. A. (1978). Comparative neurobehavioral study of a polybrominated biphenyl-exposed population in Michigan and a nonexposed group in Wisconsin. Environ. Health Perspect. 23, 199-210.

Weil, W. B., Spencer, M., and Benjamins, D. (1977). "The Effect of Polybrominated Biphenyl (PBB) on Infants and Young Children." Paper presented at the Michigan State Medical Society, Dearborn, Mich., November 8, 1977.

Whiteside, T. (1978). A reporter at large: Contaminated. New' Yorker September 4, 1978, 34-81.

Wolff, M. S., Aubrey, B., Camper, F., and Haymes, N. (1978a). Relation of DDE and PBB serum levels in farm residents, consumers, and Michigan Chemical Corporation employees. Environ. Health Perspect. 23, 177-181.

Wolff, M. S., Haymes, N., Anderson, H. A., and Selikoff, I. J. (1978b). Family clustering of PBB and DDE values among Michigan dairy farmers. Environ. Health Perspect. 23, 315-319. 